\title{
Inducible somatic oocyte destruction in response to rapamycin requires wild-type regulation of follicle cell epithelial polarity
}

\author{
TC Thomson ${ }^{1}$ and J Johnson ${ }^{\star, 1}$
}

\begin{abstract}
We show here that similar to starvation, ingestion of the bacterial product rapamycin (RAP) interferes with egg production in female Drosophila. RAP ingestion results in posterior follicle cells (PFC) in stage 8/9 egg chambers losing epithelial polarity, after which PFC invade and phagocytose the oocyte. Nurse cell apoptosis then occurs, followed by total egg chamber destruction. Knockdown of the RAP receptor FKBP12 specifically in PFC rescues oogenesis and also the laying of embryos that develop into normal offspring in flies fed RAP. Thus, somatic cells can be induced to destroy intact oocytes without a requirement for earlier oocyte compromise. Genes that control apicobasal epithelial polarity were found to be involved in egg chamber destruction. In flies bearing heterozygous mutations for discs large, merlin, or warts, PFC epithelia fail to lose polarity on RAP treatment. Embryo laying and offspring development to adulthood are rescued in all of these mutants when housed on RAP concentrations that block oogenesis in wild-type flies. The response to RAP was found to be conserved in mammals, as mouse ovarian follicles cultured in vitro with RAP show the rapid destruction of the oocyte by adjacent granulosa cells. Inducible somatic oocyte destruction is thus implicated in controlling egg survival in insects and mammals.
\end{abstract}

Cell Death and Differentiation (2010) 17, 1717-1727; doi:10.1038/cdd.2010.49; published online 7 May 2010

\section{Oocyte Development, Death, and Clearance}

Developing germ cells in females of different species are referred to as oocytes. Oocytes develop into the fertilizationcompetent eggs that support offspring production. Although the process of oocyte development (termed as oogenesis) differs greatly between insects and vertebrates, many clear developmental stages ${ }^{1,2}$ and the genes that regulate them ${ }^{3,4}$ are highly conserved.

Also conserved are the mechanisms that control oocyte death and the clearance of their corpses. In insects, ${ }^{5}$ avians, ${ }^{6}$ and mammals, ${ }^{7}$ apoptosis has been considered the primary mode of oocyte death; however, it is increasingly clear that autophagy also has a function. ${ }^{8,9}$ Studies of oocyte death have revealed that phagocytic cells engulf and destroy oocyte 'corpses.' These are the immediately adjacent somatic cells, follicle cells in insects, ${ }^{5}$ and granulosa cells in mammals. ${ }^{10}$

A critical mechanism that regulates female fertility is regulating the availability of fertilization-competent eggs. Egg production in Drosophila has been shown to be regulated by nutritional (amino-acid) availability, and endocrine and paracrine hormone signaling. ${ }^{11}$ The rate of production of new egg chambers by the germ and somatic stem cells of the germarium slows when flies are placed under starvation conditions. ${ }^{11,12}$ In addition, it has long been known that immature egg chambers (containing oocytes) degenerate and die upon starvation, within the germarium or alternatively within 'mid-stage' (stage 8/9) egg chambers ${ }^{11}$ (see Pritchett et al. ${ }^{13}$ for review). We sought to determine the relative impact of decreased 'production' versus increased 'destruction' because of nutritional withdrawal on egg laying.

During periods of compromised nutrition, activity of the target of rapamycin (TOR) kinase is decreased, leading to conserved pleiotropic downstream effects including a general inhibition of translation, ${ }^{14}$ reduced cell proliferation, ${ }^{15}$ and reduced tissue growth. ${ }^{16}$ For these reasons, experimental manipulation of TOR signaling is often used to mimic nutritional conditions. The macrolide compound rapamycin (RAP) blocks its eponymous target TOR by binding an intracellular receptor, the immunophilin FKBP12. ${ }^{17}$ RAP is produced by the actinomycete bacteria Streptomyces hygroscopicus and was discovered for its potent anti-mitotic properties when applied to fungi. It is interesting that a supposedly rare bacterial product such as RAP elicits such conserved anti-growth (and, where applicable, immunosuppressive) responses in many organisms. ${ }^{18}$ Accordingly, studies have shown that Drosophila larvae raised on food containing RAP show significant growth restriction and lessened survivability. ${ }^{19}$ We hypothesized that this model of TOR inhibition would be a similarly useful model of nutritional compromise in adult flies, and would result in consistent oocyte loss.

It has been supposed that oocyte loss occurs because of impaired intrinsic physiological and biochemical pathways within the oocyte (e.g. compromised genetic, epigenetic, or cytoplasmic oocyte competence to produce offspring when

${ }^{1}$ Division of Reproductive Endocrinology and Infertility, Department of Obstetrics, Gynecology, and Reproductive Sciences, Yale School of Medicine, New Haven, CT, USA

${ }^{*}$ Corresponding author: J Johnson, Division of Reproductive Endocrinology and Infertility, Department of Obstetrics, Gynecology, and Reproductive Sciences, Yale School of Medicine, 333 Cedar Street, FMB 329F, New Haven, CT, USA. Tel: + 203785 3162; Fax: + 203737 6118; E-mail: josh.johnson@yale.edu Keywords: atresia; epithelial polarity; germline stem cells; oocyte; ovary; phagocytosis

Abbreviations: RAP, rapamycin; dTOR, Drosophila target of rapamycin; SWH, Salvador-Warts-Hippo family

Received 30.11.09; revised 15.3.10; accepted 23.3.10; Edited by EH Baehrecke; published online 07.5.10 
fertilized). This model requires that oocyte compromise occurs first, and that signaling during degeneration and death provokes surrounding somatic cells to clear and dispose of its corpse. ${ }^{11,13}$ Instead, we show here that follicle cell invasion and phagocytosis of oocytes can be induced in the fly Drosophila melanogaster by RAP ingestion, without a requirement for oocyte degeneration or death. We further show that genes required for the establishment and maintenance of follicle cell epithelial polarity during the development are also required for RAP-induced egg chamber destruction. We show that the process can be induced by RAP in mouse follicles cultured in vitro as well, implicating a conserved process of inducible somatic oocyte destruction (ISOD) as an important regulator of oocyte survival and thus fertility.

\section{Results}

ISOD in the fly. We first placed newly eclosed virgin females with males under complete starvation conditions (agar gel plates), on food containing $10 \mu \mathrm{M}$ RAP, or on food containing ethanol vehicle. Evaluation of ovaries and egg chambers from treated flies revealed differences between the three groups (Figure 1). By day 3 of treatment, ovaries from fed flies (vehicle-treated) showed the entire continuum of oogenesis (Figure 1a). Instead, starved flies showed almost completely degenerate ovaries (Figure 1b) consistent with halted egg chamber production by germ stem cells. $^{12}$ Unlike fed or starved flies, flies housed on food containing $10 \mu \mathrm{M}$ RAP consistently showed the presence of egg chambers up to mid-stages (Figure 1c, confocal fluorescence micrograph in Figure 1d) and degenerating egg chambers were most often seen the most mature position (Figure 1d, arrowheads). These data revealed two important features of RAP's effects on oogenesis as compared with starvation. First, RAP treatment correlates with increased destruction of egg chambers at the midstages. Second, RAP treatment results in a surprising continuation of the production of new egg chambers by the stem cells of the germarium (below, Figure 2). We first characterized this RAP-specific destruction of egg chambers, then measured the consequences of a dose curve of RAP treatment on embryo laying.

In our analysis of RAP-induced egg chamber destruction (Figure 1e), we took advantage of features of degenerating mid-stage egg chambers of starved adult flies as identified by Mazzalupo and Cooley. ${ }^{5}$ In our hands, the first sign of degeneration was seen in posterior follicle cells (PFC) of stages 8 and 9 egg chambers. RAP ingestion consistently induced a loss of PFC epithelial apicobasal polarity (LOP) characterized by (Figure 1e panel i, again compare with normal intact egg chamber in top panel) PFC 'piling up' and taking on a disorganized appearance. LOP was confirmed by staining treated and control ovarioles for Armadillo protein, which was seen to redistribute in the PFC of RAP-treated flies (Supplementary Figure S1). After 'piling up,' follicle cells were seen invading the oocyte (inset of Figure 1e panel i; compare with untreated control in top panel of Figure 1e). This effect was specific to stages 8 and 9 egg chambers, as no cells in larger or smaller egg chambers showed signs of disorganization. Instead of having an obvious degenerate appearance, egg chambers exhibiting posterior LOP appeared otherwise intact, exhibiting non-degenerate nurse cell (NC) nuclei and ring canals (Figure 1e, panels i and ii).

Use of a transgenic fly line that expresses green fluorescent protein (GFP) in germ cells (see Materials and Methods) revealed uptake of the GFP by the invasive PFC (Figure 1e, panels $\mathrm{i}$ and $\mathrm{ii},{ }^{*}$, and insets), showing that phagocytosis of oocyte cytoplasm and yolk (Figure 1eii, ${ }^{*}$, and inset) by PFC begins soon after LOP. Consistent degenerative steps followed, in which NC nuclei became condensed and borders between NC, including ring canals, were disrupted beyond recognition (Figure 1eiii). Follicle cells can be seen intermixed with the degenerating oocyte and NC. Finally, an egg chamber remnant (Figure 1eiv) consisting of irregular follicle cells with central disorganized actin-rich structures (Figure 1eiv, arrowhead) and fragmented NC nuclei remains. Quantification of egg chambers undergoing degeneration in response to RAP followed to determine whether the features of degeneration were induced in a consistent manner.

Assessment of over 100 mid-stage egg chambers from flies housed on vehicle, 10 , or $20 \mu \mathrm{M}$ RAP showed that consistent proportions of degenerating egg chambers with invasive/ phagocytic PFC, but intact NC versus those with invasive PFC and NC apoptosis, were present at $40 \mathrm{~h}$ post-treatment (Figure 1f). The in vivo effects of RAP ingestion were recapitulated in an in vitro ovariole culture system (Figure 1g, after ${ }^{20}$ ). In all, we placed approximately 150 ovarioles into culture either containing $100 \mu \mathrm{M}$ RAP or vehicle. After $2 \mathrm{~h}$, $40 \%$ of stage $8 / 9$ egg chambers showed degenerative features (egg chambers with either PFC LOP alone or in combination with NC apoptosis) and as seen in vivo, whereas $8 \%$ of vehicle-treated stage 8/9 egg chambers showed signs of degeneration. The above data suggest that the features of egg chamber destruction can be placed into a stepwise, developmental order (cartoon schemes and proposed stages of egg chamber degeneration as induced by RAP are shown in Supplementary Figure S2).

We determined the consequences of RAP treatment on fertility by performing an assessment of embryo laying when newly eclosed females were mated and housed on a dose curve of the drug for 19 days (Figure 1h). Initial controls in ( $\mathrm{w}^{\star}$; pVasa-me31B-GFP) were placed with males under starvation conditions resulted in essentially zero embryos laid until flies were moved to food (Figure 1h, 'FED'). Separate mating groups were housed on food containing a dose curve of RAP (see 'Analysis of RAP uptake by flies' in Materials and Methods) up to an approximate LD20/7 days dose $(20 \mu \mathrm{M})$. RAP decreased the number of embryos laid per fly in a dosedependent manner. When moved to food lacking RAP, flies showed a substantial recovery in embryo laying (Figure $1 \mathrm{~h}$, 'DRUG REMOVED'). Importantly, separate controls were performed in which males housed on RAP for 6 days were moved to food lacking drug and mated with virgin females. No reduction in male fertility was detected (not shown). Having characterized RAP-induced oocyte destruction and its consequences for fertility, we asked whether new egg chamber production by germline stem cells continued or was arrested in the presence of the drug. 

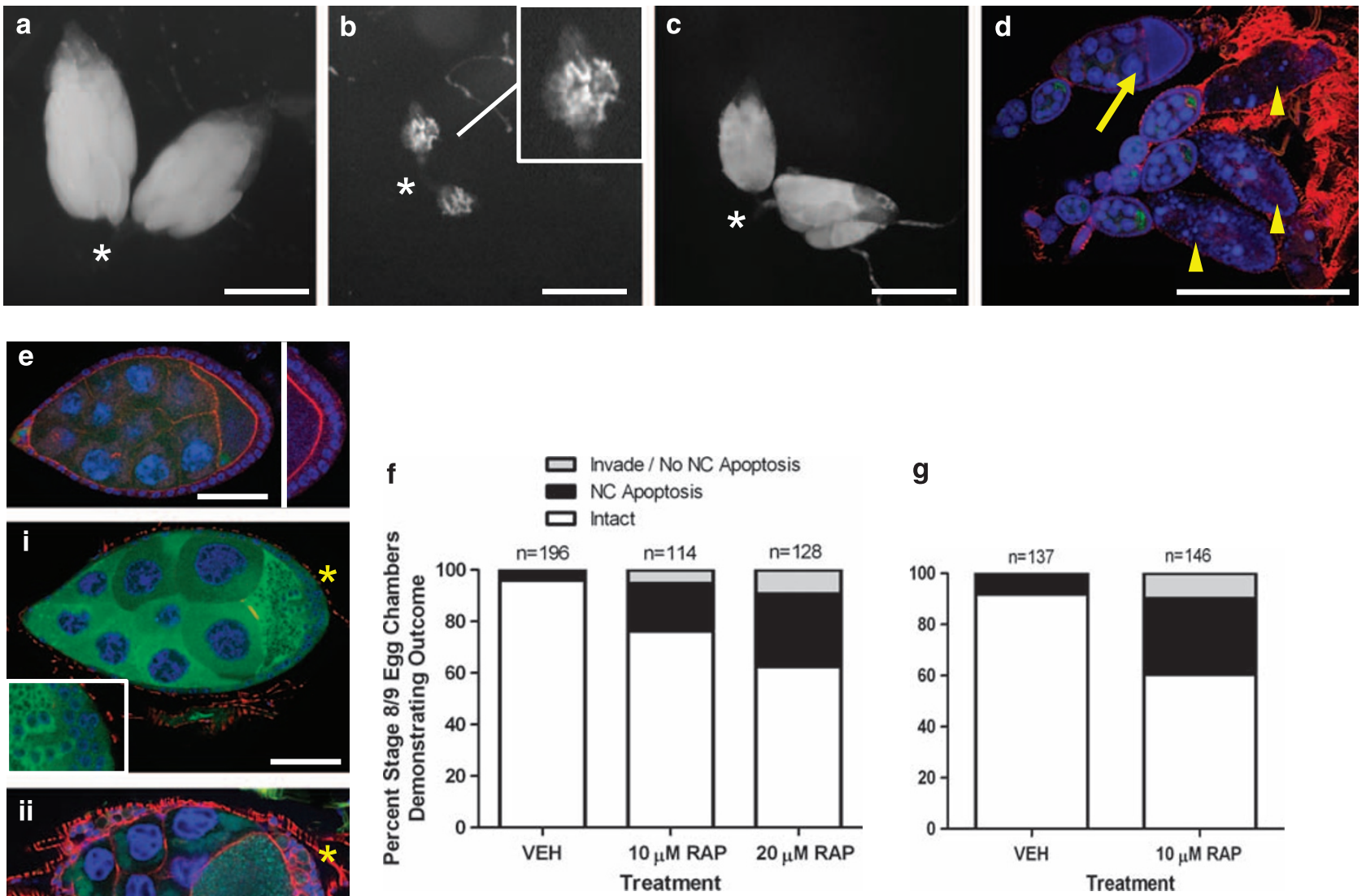

g
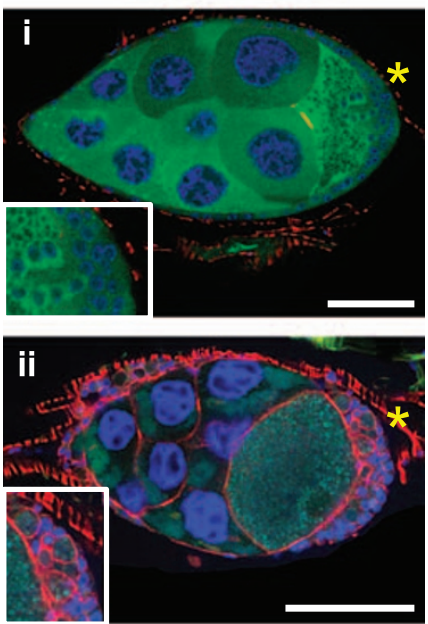

Treatment
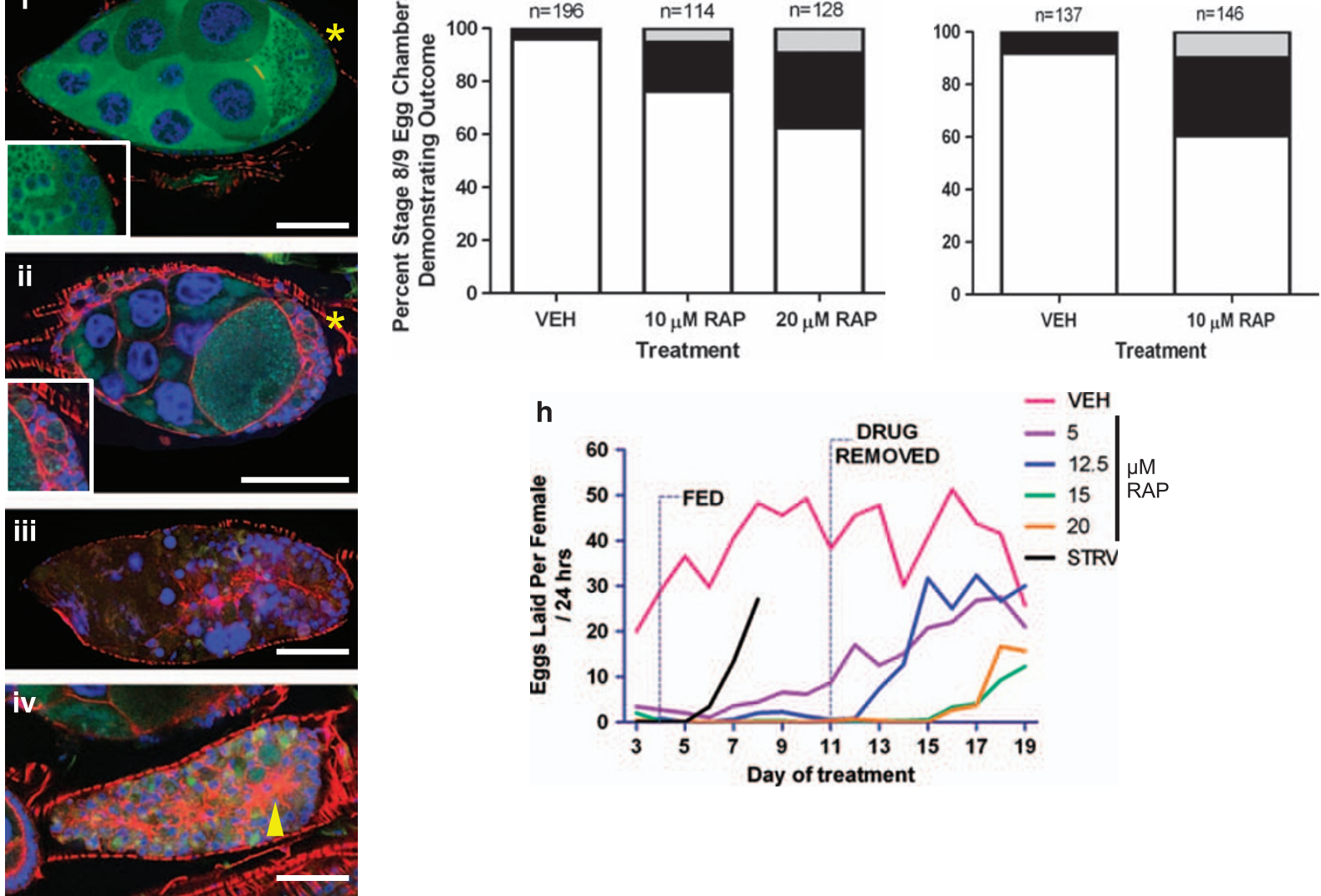

Figure 1 RAP treatment results in disrupted oogenesis in a distinct manner from starvation. (a-d) Comparison between ovaries of fed (a), starved (b), and flies fed $10 \mu \mathrm{M}$ RAP (c) positions of oviducts in a-c denoted by *, scale bars $500 \mu \mathrm{m}$. (d) Stained preparations of ovarioles from flies fed $10 \mu \mathrm{M}$ RAP reveal degeneration (arrowheads) at approximately stage 9 of oogenesis (DNA-blue, actin-red); one intact mid-stage egg chamber is present (arrow). (e) Stages of inducible somatic oocyte destruction (ISOD) as seen in w[1118]; ( $w^{*}$; pVasa-me31B-GFP) flies. DNA - blue, GFP - green, actin - red. The top image is of an intact, untreated control, and the inset shows the magnified intact polarized PFC epithelium - compare with the below numbered images of egg chambers from flies housed on food containing RAP: (i) PFCs - * lose epithelial polarity, invade the oocyte, and phagocytose oocyte contents (inset, magnified egg chamber posterior showing invasive follicle cells with internalized oocyte GFP). (ii) PFC invasion and phagocytosis continues, NC nuclei and ring canals remain intact. (iii) Follicle cells have invaded entire egg chamber, NC nuclei and ring canals degenerate. (iv) An egg chamber remnant in which follicle cells show disorganized polarity throughout; remnants are characterized by actin-rich central structures (arrowhead). Scale bars $50 \mu$ m. (f) Quantification of egg chamber degeneration in flies fed vehicle or RAP; follicle cell invasion (gray) and egg chambers showing NC apoptosis (black) increase with RAP concentration. (g) Ovarioles cultured in vitro in RAP show increased follicle cell invasion (gray) and egg chambers showing NC apoptosis (black) versus those cultured in vehicle. (h) Dose-responsive relationship between RAP concentration in food and embryo laying. Flies with designated concentration of RAP were moved to food containing no drug 'DRUG REMOVED' on day 11. Flies were also starved on plates containing hydrated agar alone ('agar plates,' see Materials and Methods) and moved to food on day 4-'FED.' Data plotted are the average embryos laid per fly in $24 \mathrm{~h}$ with a minimum of 10 flies per treatment over the course of the experiment 
VEH
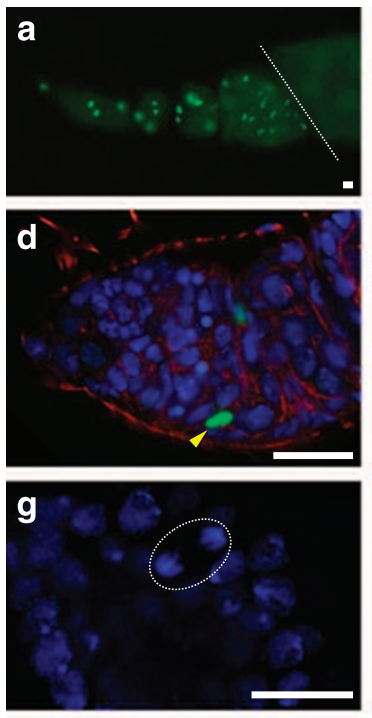

DNA
$10 \mu \mathrm{M}$ RAP
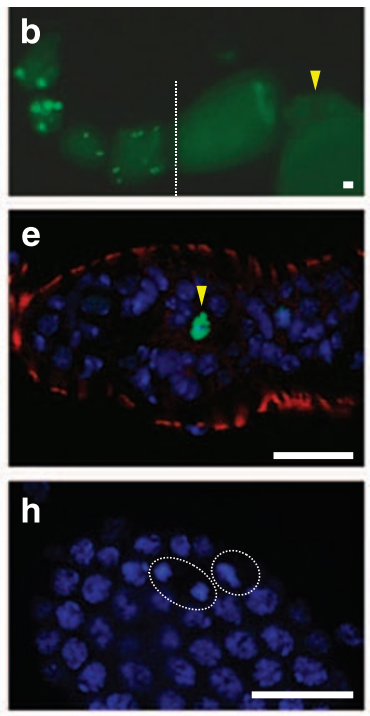

ACTIN
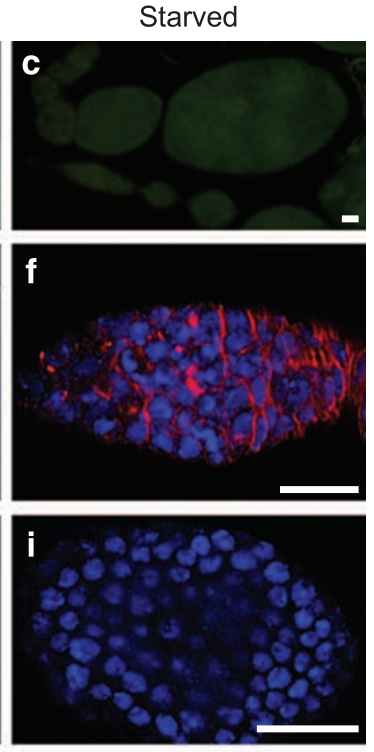

P-HH3

Figure 2 RAP ingestion (b, e, h) does not prevent the production of new egg chambers or development to mid-stages compared with vehicle (a, d, $\mathbf{g}$ ); starvation does prevent new egg chamber production $(\mathbf{c}, \mathbf{f}, \mathbf{i})$. (a-c) Immunofluorescence imaging of the mitotic marker phospho-Histone $\mathrm{H} 3$ Ser10 reveals similar numbers of mitotic cells (bright green signal) in RAP and vehicle-treated flies, lost when flies are starved; arrowhead in (b) denotes egg chamber remnant. (d-f) Mitotic events continue in the germarium of RAP-treated flies (compare with vehicle and starved). Mitotic figures ( $\mathbf{g}-\mathbf{i}$, dashed ovals) are also readily apparent using direct visualization of DNA in stage 5 egg chambers from both VEH and RAP-treated flies. Scale bars for all panels $=10 \mu \mathrm{m}$

Continued stem cell production of egg chambers in the presence of RAP. To determine potential effects of RAP on egg chamber production and growth, we evaluated mitotic figures in flies housed on 'rich food' containing $10 \mu \mathrm{M}$ RAP (Figures 2b, e, and h) or vehicle (Figures 2a, d, and $\mathrm{g}$ ). Alternatively, flies were 'starved' by housing on plates of hydrated agar (Figures 2c, f, and i; see Materials and Methods). We then examined whole-mount fluorescence immunohistochemical preparations of ovarioles for cells positive for the mitotic marker Ser10-Phosphorylated Histone $\mathrm{H} 3(\mathrm{P}-\mathrm{HH} 3$; Figures $2 \mathrm{a}-\mathrm{C})$ between the germarium and stage 6 egg chambers.

Interestingly, RAP treatment did not decrease the total number of mitotic cells relative to vehicle. We counted an average of 3.5 mitotic cells per ovariole (53 positive cells/15 ovarioles) in vehicle-treated flies (example in Figure 2a) versus 3.4 cells per ovariole (54 positive cells/16 ovarioles) in flies housed on $10 \mu \mathrm{M}$ RAP (Figure $2 \mathrm{~b}$ ). Consistent with an earlier report, ${ }^{12}$ starved control flies had reduced mitotic activity, having only one mitotic cell per ovariole (22 positive cells/22 ovarioles; Figure 2c). When considering mitotic activity within the germaria of the above ovarioles only, vehicle, RAP-treated, and starved flies showed 9, 7, and 1 germaria with one or more positive cells (examples shown in Figures $2 \mathrm{~d}-\mathrm{f}$ ), respectively. $\mathrm{P}-\mathrm{HH} 3$ staining was recapitulated by assessment of mitotic chromosomes using DNA staining alone (Figures $2 \mathrm{~g}-\mathrm{i}$ ). Thus, new egg chamber production and growth in early stages continues nearly apace when RAP is applied. This is counter to the effects seen in most cell types studied, which instead favor slowed proliferation when TOR kinase is specifically inhibited. ${ }^{19,21}$ In our studies, only starvation correlated with ovariole cell cycle arrest, consistent with earlier findings. ${ }^{12}$

Inducible PFC destruction of egg chambers. Having characterized the effects of RAP on oogenesis and embryo laying, our next objective was to determine whether the effects of RAP treatment was indeed dependent on the inhibitor binding its known receptor, FKBP12. First, we determined the expression of FKBP12 in stage 8 egg chambers (Figure 3a). FKBP12 was found to be expressed in follicle cells and NCs, with lower levels detected in the oocyte. We then generated fly lines that express RNAi targeted to FKBP12 in follicle cells in different regions of the egg chamber. We hypothesized that knockdown of FKBP12 in PFC would inhibit egg chamber destruction, perhaps leading to lessened LOP, or lessened follicle cell invasion into the oocyte. Accordingly, we hypothesized that controls using an anterior follicle cell (AFC)-specific promoter would not prevent the process.

Our hypothesis proved correct, as flies bearing a PFCspecific GAL4 driver and UAS-FKBP12 RNAi (example of FKBP12 expression knockdown in PFC shown in Figure 3b, additional examples in Supplementary Figure S3) continued to lay embryos when housed with males on $10 \mu \mathrm{M}$ RAP (Figure 3c). We counted embryos laid per fly from our experimental and control strains on each day post-eclosure for 6 days. To ensure that we accounted for basal strain laying differences, we calculated the percentage of embryos laid versus vehicle-treated same-strain controls. When raised on the $10 \mu \mathrm{M}$ concentration of RAP that obliterated embryo laying by wild-type flies (Figure 1h), flies expressing an AFC-specific 

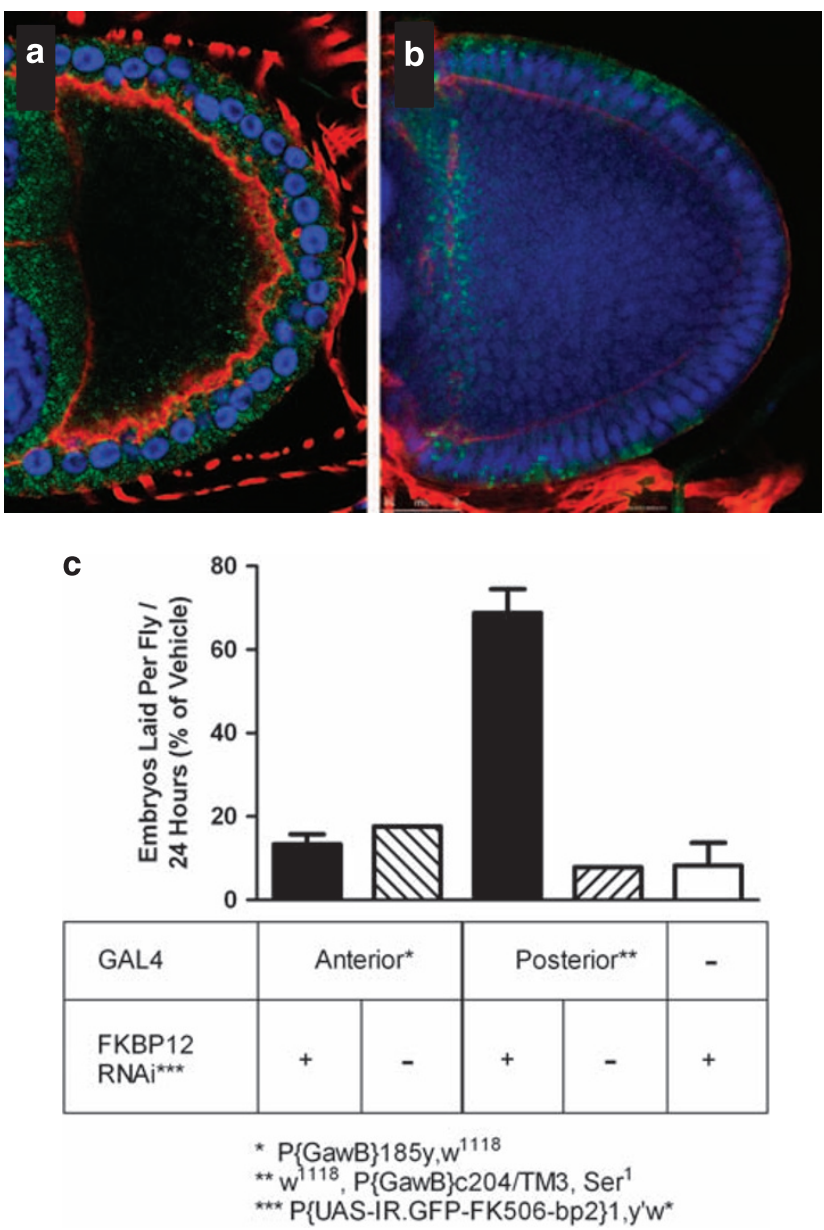

Figure 3 Follicle cell expression of the RAP receptor FKBP12 is required for the induction of egg chamber destruction by RAP. (a) Fluorescence immunodetection of FKBP12 (green; DNA - blue, actin - red) in wild-type mid-stage egg chamber reveals expression in follicle and NCs, with reduced expression in the oocyte. (b) Representative image of FKBP12 expression in PFC knockdown fly line (see table in c, Posterior GAL4, RNAi ' +'). (c) Knockdown of FKBP12 specifically in PFCs using a posterior-specific GAL4 driver ${ }^{(*)}$ rescues egg laying on flies housed on food containing $10 \mu \mathrm{M}$ RAP. Note that flies expressing FKBP12 RNAi using an AFC GAL4 driver $\left(^{*}\right)$ neither lay appreciable embryos versus controls when housed on RAP, nor does expression of the UAS-RNAi alone. Data shown are from the 5 th day of continuous treatment post-eclosure

GAL4 driver and UAS/FKBP12 RNAi, also flies expressing the driver or UAS/RNAi alone, and flies expressing the PFCspecific driver and RNAi instead showed a significant rescue of embryo laying (Figure 3c). As the GAL4/UAS system used is known to be effective in somatic cells, but not the germline, ${ }^{22,23}$ and RNAi has been shown to be non-functional in oocytes until much later in oogenesis (stage $14,{ }^{24}$ ), it is likely that laying indeed results from somatic follicle cell effects and not from any unanticipated knockdown effects within oocytes.

Beyond the immediate result, the demonstration that the RAP receptor is required in follicle cells for the drug's effects, this experiment allowed us to address a long-standing question in reproductive biology. Egg chamber death in insects and ovarian follicle death - termed as atresia - in mammals have been studied for many years. Oocyte loss in insects and mammals has been discussed in terms of a supposed function in 'quality control,' in which oocytes that have diminished genetic, epigenetic, or cytoplasmic integrity die and are then cleared by surrounding somatic cells. However, it has never been conclusively determined whether intrinsic oocyte damage is in fact a prerequisite for oocyte loss. We used this PFC-specific FKBP12 knockdown system to address this question as follows. If maternal RAP damaged oocytes during development, even though embryo laying was rescued in the knockdown flies, a fraction of damaged embryos would be incapable of developing into adults. To test this, we moved laid embryos from PFC FKBP12 knockdown flies to fresh standard fly food containing no RAP (to avoid the problem of RAP preventing larval development as per Zhang et al. $^{19}$ ). We found that 108 of 120 embryos hatched and developed into normal adults (by way of comparison, 112 of 120 wild-type control embryos from untreated mothers that were similarly moved to fresh fly food developed into adults). This result shows that maternal RAP treatment does not compromise an oocyte's (e.g. the germ compartment's) ability to complete oogenesis and give rise to offspring. Instead, we have shown that oocyte-extrinsic cues can induce somatic follicle cells to destroy oocytes that would otherwise be capable of giving rise to offspring.

As FKBP12 expression is required within PFCs for the induction of mid-stage egg chamber destruction by RAP, and normal oogenesis and offspring production occur in our FKBP12 PFC knockdown model, somatic follicle cell-specific effects are both necessary and sufficient to carry out oocyte destruction and halt oogenesis and fertility. We thus refer to this process as ISOD to make a clear distinction with models of oocyte loss that involve earlier or concurrent oocyte damage.

Although we were able to define ISOD as an ordered process with consistent consequences, the genes that execute oocyte destruction within follicle cells remained unknown. We hypothesized that genes known to control follicle cell development and/or follicle cell epithelial polarity were required for the initiation or execution of the process of egg invasion and destruction.

ISOD requires intact signaling by genes involved in the control of PFC tissue size and epithelial polarity. We engaged in a candidate gene approach to test whether known regulators of follicle cell layer development are also required for ISOD. We first confirmed that egg laying was halted in wild-type Canton-S flies and the me31b/GFP controls used earlier when fed RAP. To account for the action of RAP on the TOR signaling pathway, we examined embryo laying by Drosophila target of rapamycin (dTOR) mutant flies. Zhang et al. $^{25}$ showed that strong mutations in dTOR kinase exhibit growth retardation and significantly compromised oogenesis. We hypothesized that compromised oogenesis would occur even in flies heterozygous for a strong loss-of-function $d T O R$ allele, and that this would be exacerbated by RAP treatment.

Next, we evaluated members of the Salvador-Warts-Hippo (SWH) network of proteins known for their functions in the control of cell growth, tissue size, ${ }^{26}$ and oocyte axis determination $^{27,28}$ through action within the PFC. The tumor 
suppressor Merlin/Neurofibromin 2 (Mer) has recently been proposed to be an SWH network participant, ${ }^{29}$ and, through the regulation of Warts (Wts) and Hippo, has also been shown to be required to establish proper oocyte axis formation. ${ }^{30} \mathrm{Wts}$ and two unique alleles of $\mathrm{mer}^{31}$ were tested here. Scribble (scrb) and discs large ( $d l g)$ are among the genes that control the polarity of the follicle cell layer ${ }^{32,33}$ by participating in junctional complexes between epithelial cells, and flies heterozygous for these genes were also tested. Last, we tested whether Draper (drpr), a gene that acts as a receptor for dead and dying cells during the development of the nervous system, ${ }^{34}$ is required for oocyte destruction. Drprhas also been shown to function within cell competition models, in which cells of abnormal size (e.g. Minute mutations) die and are targeted for destruction. ${ }^{35}$ Here, we hypothesized that drpr would be required to target and engulf oocytes in a similar manner.

Our preliminary screen was executed as follows. Embryo laying of mutant and wild-type flies was monitored every $24 \mathrm{~h}$ for 6 days in the presence or absence of RAP, or under starvation conditions. First, examination of ovaries and ovarioles was performed on untreated flies from all strains and we found that there was no significant difference between the basal rate of egg chamber degradation in any strain when compared with Canton-S flies. Of all strains, only wts had a significantly altered rate of embryo laying (laying approximately half the embryos per day as the other strains). The average number of embryos laid by all other strains on the first 6 days post-eclosure was indistinguishable from Canton-S flies (data not shown). In the presence of $10 \mu \mathrm{M}$ RAP, Canton$S$ and me31b-GFP flies laid a small fraction of eggs compared with vehicle-only controls (Figure $4 \mathrm{a}$, data graphed as a percent embryos laid of vehicle-treated controls at days 3-6 of treatment). $d T O R /+, s c r b /+, \mid g l /+$, and $d r p r /+$ (not shown, see below) flies all showed the same drastic loss of oogenesis as found with wild-type flies.

In striking contrast, flies heterozygous for functional alleles of several of our candidate genes rescued embryo laying in flies fed RAP (Figure 4a). Heterozygous dlg (null allele $\mathrm{dlg}^{\prime v 55 /+)}, \mathrm{merl}+$, and wts/ + mutants showed significantly increased embryos laid per fly versus wild-type controls and controls for genetic background (see Materials and Methods). Examination of whole ovary pairs (representative mer ${ }^{4}$ ovaries from flies raised on $10 \mu \mathrm{M}$ RAP shown in inset; Figure 4a) and ovarioles from treated mutant flies showed the expected rescue of oogenesis; these mutants had vastly fewer degenerating egg chambers on RAP than controls. To test whether flies showing rescued oogenesis were simply showing an overall increased resistance to RAP, $20 \mu \mathrm{M}$ was applied, a dose that we found is the approximate LD20/7 days for all wild-type and mutant fly lines tested. We found that even at this sometimes-lethal dose, surviving $\mathrm{dlg} /+, \mathrm{mer} /+\left(\mathrm{mer}^{4} /+\right.$ at $20 \mu \mathrm{M}$ shown), and wts/+ flies laid significant numbers of embryos. The similar lethality of this dose across strains suggests that individual mutants are not more resistant to high concentrations of RAP in food. Further, control western blots (Figure $4 \mathrm{~b}$ ) revealed that the dTOR kinase substrate p70S6kinase is phosphorylated at essentially wild-type levels in untreated $\mathrm{dlg}^{\mathrm{lv55} /+}, \mathrm{mer} /+$, and wts/+ mutant flies, and its phosphorylation is greatly diminished in RAP-treated flies of each strain. This suggested that the reduced ISOD seen in mutants is unlikely to be a result of de-regulated TOR signaling as a result of each mutation.

We performed additional controls to confirm the genetic basis of rescued embryo laying in flies fed RAP. We controlled for the genetic background of $\mathrm{dlg}^{\mathrm{lv55}}$ and $\mathrm{mer}^{4}$ by generating heterozygous flies in a wild-type Canton-S background. Both outcrossed control strains showed a significant rescue of egg laying on RAP similar to their parent strains (data not shown). Further, comparison between two alleles of $\mathrm{mer}^{31}$ showed that flies heterozygous for a weaker allele, mer3, had a delayed rescue (compare $\mathrm{mer}^{3} /+$ and $\mathrm{mer}^{4} /+$ on day 3 of embryo collection; Figure 4a) and fewer average embryos laid when compared with flies heterozygous for a stronger allele, $\mathrm{mer}^{4} /+\left(\mathrm{mer}^{3}\right.$ results in an amino-acid substitution, $\mathrm{M} 177 \mathrm{I}$, although $\mathrm{mer}^{4}$ is a premature stop codon mutation, Q170STOP). We also tested flies heterozygous for a temperature-sensitive allele of $d l g\left(d l g^{h f}\right)$ with near-wild-type function at permissive temperature $\left(18^{\circ} \mathrm{C}\right)$ and hampered function at restrictive temperature $\left(25^{\circ} \mathrm{C}\right)$. We found that both heterozygous flies bearing the null allele $d^{\prime} g^{1 / 55}$ and, separately, the temperature-sensitive allele $d^{\prime g} g^{h f}$ at restrictive temperature robustly rescued embryo laying, whereas $d l g^{h f} /+$ flies raised at the permissive temperature resulted in fewer embryos laid.

In light of the rescue of oogenesis, embryo laying, and offspring production in epithelial polarity mutants, we tested whether these mutations also rescue oogenesis under conditions of nutritional compromise. We housed flies under complete starvation conditions (agar plates alone), partial starvation conditions (grape juice agar plates ${ }^{5}$ ), or on 'rich food (grape juice agar with added yeast ${ }^{5}$ ).' As seen in wildtype and me31b-GFP flies (Figure 1h, 'STRV'), egg chamber production by epithelial polarity mutant flies was completely blocked when they were housed on agar plates lacking juice (data not shown). Comparison between rich food and partial starvation revealed a vast reduction in embryo laying in flies housed on juice agar in heterozygous $\mathrm{dlg}^{\mathrm{lv55} /+}, \mathrm{mer}^{4} /+$, and $w t s /+$ flies (Figure $4 \mathrm{c}$ ). Of the mutants, only the dlg allele showed slight but significantly increased embryo laying under partial starvation conditions versus 'wild-type' (me31b-GFP) controls. Here again, we tested $\mathrm{dlg}^{\mathrm{lv55} /}+$ and $\mathrm{mer}^{4} /+$ outcrossed into a Canton-S background, and found nearly identical effects correlating with each mutation (not shown). Analysis of ovaries, ovarioles, and embryos from mutants raised under the specified conditions followed. Although embryo laying was partially rescued in the noted mutants, we did detect differences in egg chamber degeneration in mutants raised in the presence of RAP versus the process seen in wild-type flies (Figure 1e). Degenerating egg chambers from $\mathrm{dlg}^{\mathrm{lv55} /}+$ (Figure 4d), $\mathrm{mer}^{3} /+$ (Figure 4e), and $\mathrm{mer}^{4} /+$ (Figure 4f) flies are shown - compare with "wild type' (me31b-GFP) example (Figure 1eii and iii). Degenerating mid-stage $d g^{\prime v 55} /+$ egg chambers were characterized by intact and non-invasive PFC epithelia surrounding apoptotic NC nuclei. In keeping with embryo laying data, comparison between the two alleles of mer shows that the weaker allele $\left(m^{2} r^{3} /+\right.$; Figure 4e) correlates with increased follicle cell disorganization relative to a stronger allele $\left(\mathrm{mer}^{4} /+\right.$; Figure 4f). ${ }^{31}$ Although PFC were more disorganized in 


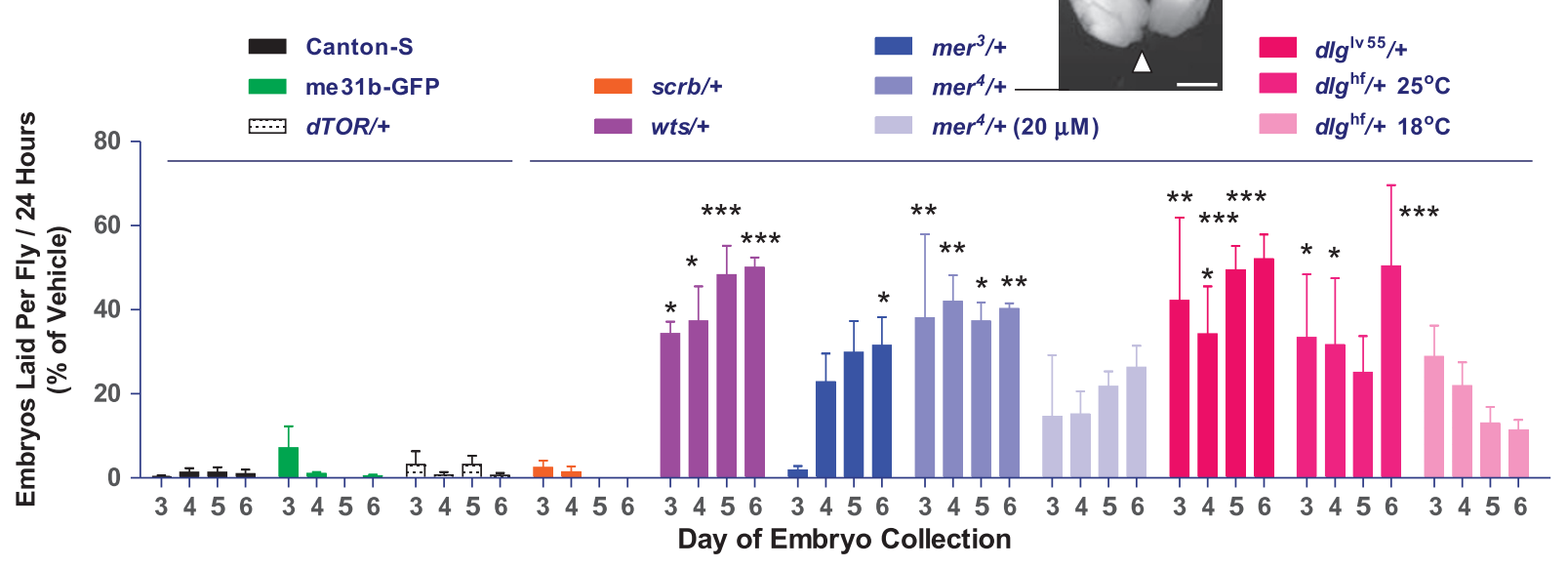

b

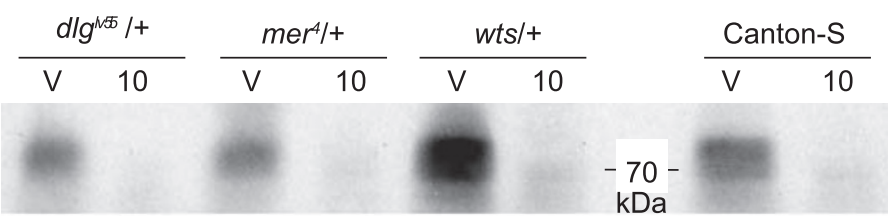

GAPDH
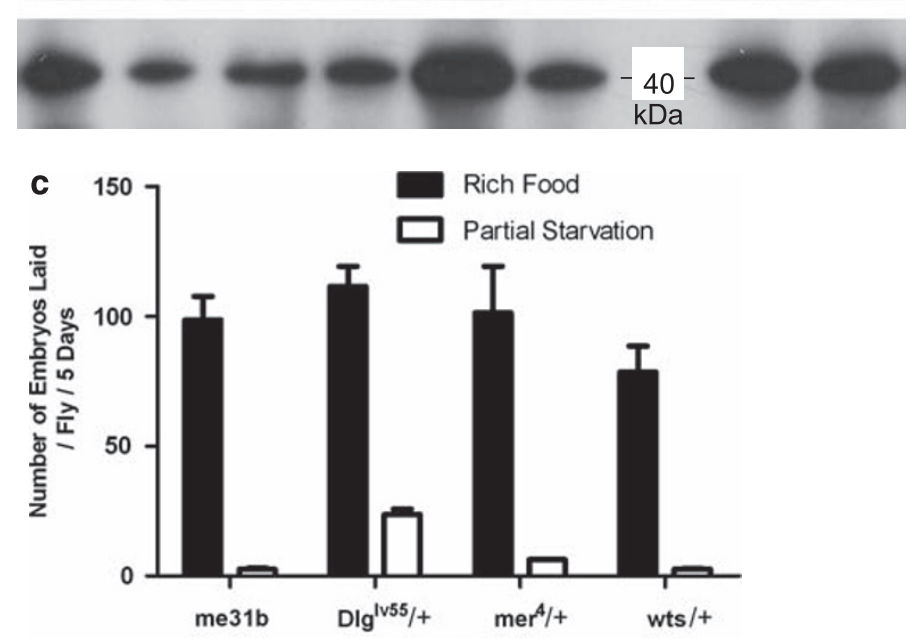

Fly Strain
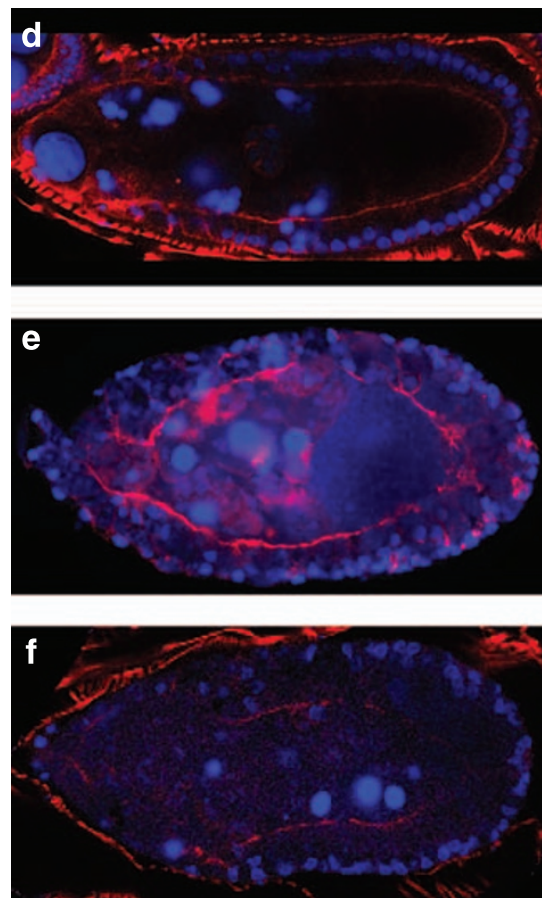

Figure 4 Mutations in genes known to control epithelial polarity rescue embryo laying in RAP-treated flies. (a) Flies heterozygous for mutations in dlg, mer, and wts continue to lay embryos on $10 \mu \mathrm{M}$ RAP (data graphed as a percent of vehicle-treated intra-strain controls). Similar to wild-type Canton-S (and w*; pVasa-me31B/GFP) flies, $d T O R /+$ and $S c r b /+$ mutants do not lay appreciable numbers of embryos on RAP. Statistical significance was determined comparing all strains to Canton-S flies (at each corresponding day of embryo collection) by two-way ANOVA with Bonferroni post hoc test; asterisks denote $P$-values where ${ }^{*}=P<0.05,{ }^{* \star}=P<0.01$, and ${ }^{\star \star *}=P<0.001$. Inset shows representative whole ovary pair from heterozygous mer4 mutant flies fed $10 \mu \mathrm{M}$ RAP (position of oviduct denoted by arrowhead, scale bar $500 \mu \mathrm{m}$ ), compare with Figure 1d. (b) dTOR is active at approximately wild-type levels in heterozygous dlg, mer, and wts mutant female flies as measured by phosphorylation of p70S6-kinase; RAP treatment reduces P-p70S6K phosphorylation in mutant flies as seen in wild-type flies. ' $V$ ' denotes vehicle-treated flies and ' 10 ' denotes flies housed on food containing $10 \mu \mathrm{M}$ RAP. (c) Although no fly strains examined lay embryos under complete starvation conditions, $d l^{\prime l v 5} /+$ flies show a slight but significant $(P<0.01$, Student's $t$-test) rescue of embryo laying under partial starvation conditions. (d-f) Degenerating egg chambers from mutants reveal function(s) in remodeling of follicle cell epithelium on RAP treatment. Although NC apoptosis occurs, follicle cells from flies heterozygous for dlg (d), and the mer4 allele (f) fail to become disorganized and do not invade the germline. Egg chambers from mer3 heterozygotes show LOP, but still do not invade the germline (e)

$m e r^{3} /+$ than $m e r^{4} /+$ egg chambers, no oocyte invasion by PFC was seen in these mutants. These data further show that gene dosage of epithelial polarity genes controls the initiation and execution of ISOD in response to RAP.

We interpreted these data to mean that the induction of PFC LOP is required for the successive steps of ISOD, and when not induced efficiently (as in the case of the mutants that rescue egg laying in the presence of RAP), the process is bypassed, and oogenesis continues. It is striking that two copies of $d l g$, mer, and wts are required to induce and execute ISOD (comparison between mutant phenotypes summarized in cartoons in Supplementary Figure S4). These mutants reveal that genes involved in the establishment and maintenance of epithelial polarity are also required for the 
regulated loss of polarity and subsequent oocyte invasion of PFC seen during induced oocyte destruction.

In contrast, flies mutant for the engulfment receptor $d r p r$ maintained on RAP engage in stage 8/9 ISOD in a manner that approximates that seen in wild-type flies. Drpr mutants show all of the hallmarks of ISOD. Interestingly, drpr mutant ovarioles were found to contain an elevated number of egg chambers with apoptotic nuclei at every stage of development (Thomson and Johnson, unpublished), suggesting that the basal rate of clearance of dead and dying cells in wild-type egg chambers is under-appreciated. This is in keeping with the function of Drpr in the recognition and clearance of dead, damaged, and dying neurons, ${ }^{34}$ and the observation that Minute cells die before being recognized, surrounded, and cleared during cell competition. ${ }^{35}$ However, as there is no apparent requirement for Drpr for oocyte invasion and destruction by follicle cells, the likelihood that oocyte damage and/or death signals need not precede invasion was further supported. Combined with the rapid induction of the process (e.g. from treatment to egg chamber remnant in approximately $2 \mathrm{~h}$ ), and the follicle cell-specific requirement of FKBP12 for induction and execution, ISOD seems to represent a novel mode of cell death that involves regulated epithelial polarity and activation of a phagocytic program to destroy an adjacent cell.

\section{Consequences of RAP treatment on offspring} development. We went on to test the potential of the rare wild-type embryos laid by RAP-treated wild-type females or the comparatively abundant embryos laid by RAP-treated heterozygous mer, dlg, or wts flies to hatch and develop to adulthood. As mentioned, male reproductive function was not affected by RAP. Also as mentioned, RAP treatment of larvae (phenocopied by TOR mutations) compromises growth, development, and survival to adulthood. ${ }^{19,36} \mathrm{We}$ thus moved laid embryos from treated flies of these genotypes to food containing no drug and found that embryos laid by RAP-treated mothers were grossly normal. However, hatching and development to adulthood was compromised in offspring of treated mothers as follows: $7 \%(4 / 55)$ of Canton-S, 40\% (72/180) of $\mathrm{mer}^{4} /+$, 25\% (20/120) of $w t s /+$, and $32 \%(50 / 157)$ of $\mathrm{dlg}^{\mathrm{lv55} /+}$ hatch and survive to adulthood, respectively. Compared with the approximately $90 \%$ hatching and survival rate of FKBP12 PFC knockdown offspring (Figure $3 \mathrm{c}$ and above) this suggests that although oogenesis is rescued, defects in offspring can arise when RAP is present and ISOD does not occur or is not executed correctly.

ISOD is conserved in the mouse. As mentioned, mammalian $^{7}$ and avian $^{6}$ follicles bearing oocytes die in a process referred to as atresia. Atresia culminates in the regression of the follicle in a little understood process in which a fraction of live granulosa cells engulfs dying and dead granulosa cells and the oocyte, ${ }^{10}$ resulting in a degenerate remnant. Earlier studies in our laboratory ${ }^{37}$ showed that RAP treatment of preantral (e.g. immature) mouse follicles cultured in vitro resulted in compromised follicle growth because of the inhibition of granulosa cell proliferation, without inducing granulosa cell death.
Our Drosophila results spurred us to repeat those experiments and carefully assess the disposition of oocytes and follicles after culture in RAP.

As expected, vehicle-treated follicles showed significant granulosa cell proliferation $48 \mathrm{~h}$ after culture in media containing the gonadotrophin FSH (compare freshly isolated/uncultured follicle in Figure $5 a$ to same follicle cultured in vehicle, Figure 5b). We found that RAP treatment caused three dosedependent effects on cultured follicles. First, in keeping with the effects seen in flies, oocytes were displaced by granulosa cells in a manner that rendered the oocytes unrecognizable (Figure 5c). A similar loss of oocytes can be induced by omitting FSH from culture media (Figure $5 d$ ). Second, follicles are seen in which the granulosa cell layers did not proliferate, but remain intact (Figure 5e). Treated follicles lacking oocytes or that did not grow were confirmed as viable using a biochemical assay (after Yaba et al., ${ }^{37}$ data not shown), showing that the destruction of oocytes in these mouse follicles was again unlikely to be a passive degenerative process as in flies. Last, we found that either FSH omission or treatment with $200 \mathrm{nM}$ RAP resulted in a fraction of follicles that lost spherical integrity and 'plated down' onto the culture surface (Figure 5f). This result is reminiscent of the LOP seen in treated flies and ovarioles and warrants further study as regards the 'polarity' of granulosa cell layers. These data are summarized in the graph in Figure $5 \mathrm{~g}$.

These data suggest that at least in the case of RAP treatment, that oocyte invasion and destruction by adjacent somatic cells is conserved in the mouse during the early window of ovarian follicle growth. It remains to be seen whether somatic-specific effects are sufficient to cause oocyte destruction as seen in the fly. As orthologs of genes relevant to epithelial apicobasal polarity and growth control have been detected within mouse ovarian follicles (including scribble and discs large $\left.{ }^{38}\right)$, it is possible that the genes involved in initiating and executing oocyte destruction are also conserved in mammals.

\section{Discussion}

ISOD is a unique mechanism that controls oocyte survival. We, therefore, show that the response to the bacterial product RAP is unique from that of complete starvation (Figures $1 \mathrm{a}-\mathrm{e}, \mathrm{h}$ and 2 ), and that this response is follicle cell specific (Figures 3; Supplemetary Figure S3). We determined that the ovaries of $d T O R /+$ flies neither contain an increased basal rate of degrading egg chambers, nor does heterozygosity for this allele results in reduced embryo laying compared with wild-type flies, showing that reduced gene dosage of the RAP target kinase alone does not result in oocyte destruction. Consistent with this, larvae heterozygous for a dTOR mutant allele also do not show slowed growth or development to eclosure when untreated. However, the addition of RAP to larval food resulted in an additive effect in which $d T O R /+$ larvae developed to eclosure days slower than wild-type larvae. ${ }^{19}$ In our studies, $d T O R /+$ flies fed RAP laid almost no embryos, just as found with wild-type flies (Figure 4a). Further, the induction of oocyte destruction by RAP was shown to require 

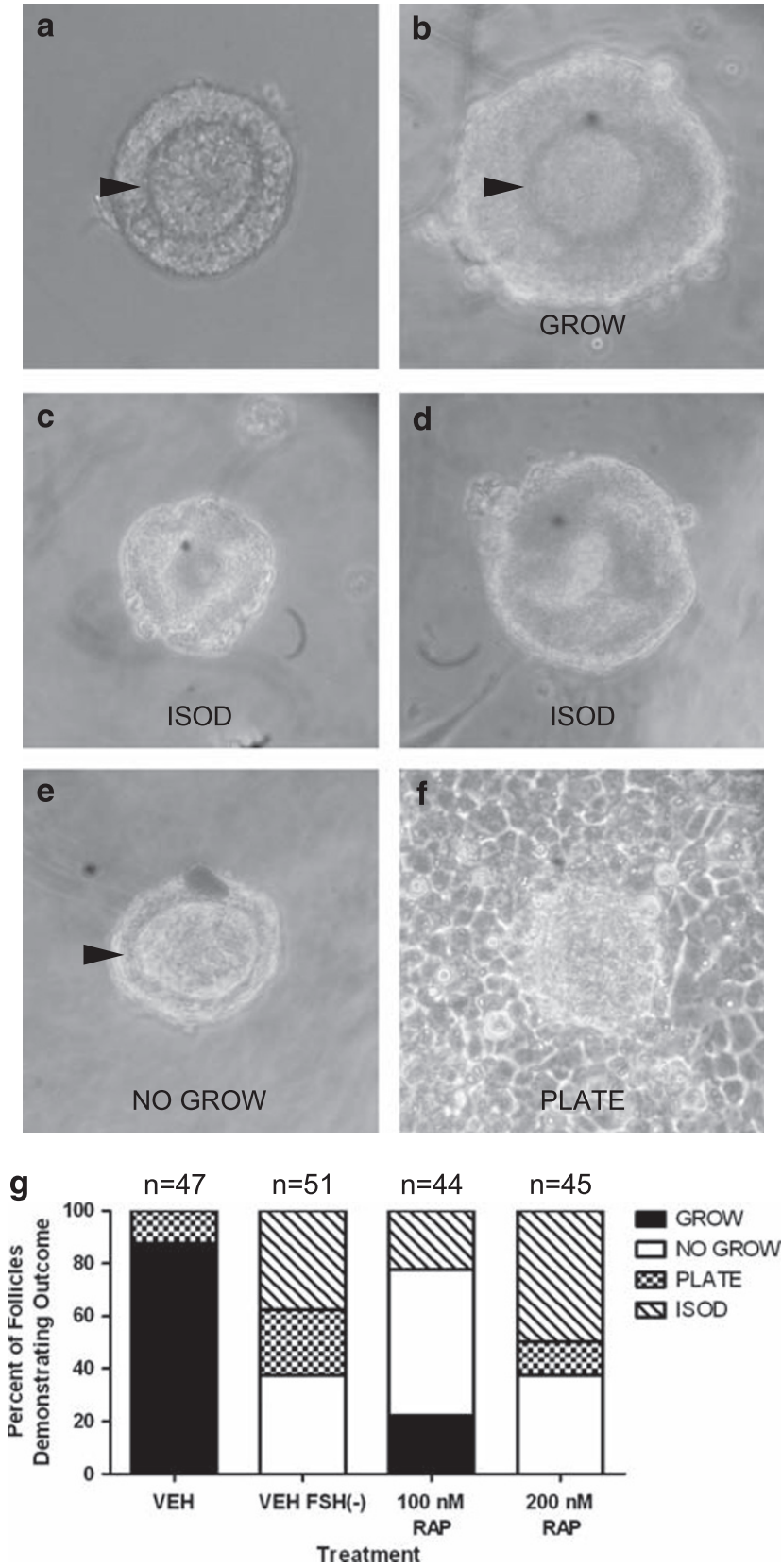

Figure 5 Somatic (granulosa) cell oocyte destruction is induced by RAP treatment during in vitro mouse follicle culture. (a) Freshly isolated mouse follicle; the border of the centrally located oocyte is sharp (arrowhead). (b) Follicle shown in (a) cultured for $48 \mathrm{~h}$ in vehicle (ethanol). Proliferation of the granulosa cell layers has occurred and the oocyte remains centrally located (arrowhead); denoted 'GROW.' (c) Example of follicle cultured in media lacking FSH; the oocyte has been invaded by the granulosa cells 'ISOD.' (d) Example of follicle cultured in the presence of $100 \mathrm{nM}$ RAP; again, the oocyte has been invaded by granulosa cells. (e) Example of follicle in which the oocyte is intact, but the granulosa cell layers fail to proliferate in the presence of $100 \mathrm{nM}$ RAP 'NO GROW.' (f) Example follicle cultured in $200 \mathrm{nM}$ RAP in which the spherical follicular structure has 'plated down' and cells have spread on the culture plastic, 'PLATE.' (g) Graph summary of outcomes for approximately 45 follicles cultured in vehicle (VEH), in media containing 100 or $200 \mathrm{nM}$ RAP, or, in media containing VEH, but where FSH was omitted

FKBP12 expression (Figure 3) and to correlate with significantly reduced phosphorylation of the dTOR substrate p70-S6 kinase (Figure 4b). These data support the RAP-sensitive TOR complex 1 (TORC1) as the inhibited target that results in ISOD, but alterations in PFC polarity and position during invasion suggest that inhibition of dTOR within the actin cytoskeleton regulating $\mathrm{TORC}^{39}$ may also be involved.

We further show that genes required for the establishment and/or maintenance of proper PFC epithelial polarity are also required for the induction of oocyte destruction (Figure 4) during RAP treatment. As flies that bear a single copy of $d l g$ fail to initiate oocyte destruction (e.g. do not lose follicle cell polarity; see Figure 4d) and flies heterozygous for mer (Figures $4 \mathrm{e}$ and $\mathrm{f}$ ) or wts show disorganized PFC organization after RAP treatment, but not PFC invasion or phagocytosis, the successive stages of oocyte destruction correlate with two intact copies of these genes. Prevention of the induction of ISOD not only rescues the destructive process seen in mid-stage egg chambers, but can entirely rescue the production of normal mature eggs and normal offspring in certain cases.

These data are consistent with earlier studies, in particular those identifying the impact of nutritional compromise on egg chamber production ${ }^{11,12}$ and destruction. ${ }^{5}$ We have added to this body of work in the following ways. First, the use of RAP both reveals its unique effects on oogenesis and also allows us to place the successive stages of egg chamber destruction in temporal order. Second, we have identified genes responsible for the initial step in ISOD, the loss of PFC epithelial polarity. Last, assessment of embryo laying and offspring production in flies shows that altering the control of epithelial polarity in PFC can rescue not only oogenesis, but also offspring production (below). Further analysis is warranted to determine the effects of dTOR pathway mutations ${ }^{25}$ and specific dietary conditions (amino-acid and/or sugar availability) and hormonal (ecdysone, juvenile hormone, sex peptide) stimuli ${ }^{11}$ on egg production and destruction.

ISOD response is distinct from models of follicle cell tumorigenesis. The surprising requirement for genes involved in follicle cell epithelial polarity for ISOD induction reveals new information about Dlg, Mer, and Wts function. Zhao et al. $^{32}$ showed that increasingly strong discs large mutations result in increased invasion of follicle cells into the germ compartment of egg chambers. This invasion was enhanced by concurrent heterozygosity for $l g l$, wts, and scrb. In the case of double mutants, follicle cell proliferation was seen to be strongly enhanced resulting in large follicle cell tumors in mid-stage egg chambers and beyond. In no case did we see follicle cell proliferation during stage 8/9 ISOD. Importantly, the weakest example(s) of dlg mutants showed no follicle cell invasion, ${ }^{32}$ in contrast to our results when those same flies were housed on RAP or were partially starved. Also of interest is our finding that scrb and $\mathrm{lgl}$ mutants did not rescue oogenesis in the presence of RAP, unlike mer and wts. The combinatorial action of these genes during tumorigenesis is, therefore, different than their functions in the control of mid-stage egg chamber destruction.

These results speak of an interesting gene dosagedependent mechanism, in which homozygosity for the above epithelial polarity genes is required for a regulated loss of 
polarity (involving rearrangement of the oocyte and follicle cell actin cytoskeleton) and the activation of a phagocytic program in follicle cells on the ingestion of RAP. That single distant, unrelated, and 'mild' (e.g. do not act as dominant alleles under control circumstances) mutations can function in a dominant manner in the presence of RAP bears further study - it may be that other genes have the same dosage requirement for their action in follicle cells and in other epithelia.

The loss of epithelial polarity induced by RAP was also reminiscent of the work by Mirouse et al., ${ }^{40}$ who showed that 'energetic stress' (in their model, reduced sugar availability) resulted in an LOP in follicle cell clones mutant for the AMPK catalytic subunit ampk $\alpha$. As AMPK is known to act upstream of TOR kinases, our data are consistent. However, in contrast to that work, in which LOP was detected in follicle cell clones in many stages of egg chamber development, we only detected altered follicle cell organization in stages 8 and 9 egg chambers. This suggests that additional factors maintain epithelial organization in egg chambers outside of the midstages during RAP ingestion.

Implications for female fertility. Our data also preliminarily support a novel example of cellular destruction of a neighboring cell, limited to a specific developmental window of time and location (mid-stage egg chambers). The rapid execution time, its dependence on somatic follicle cell FKBP12 function, and our lack of detection of oocyte death when flies, ovarioles, or mouse follicles are treated with RAP invokes a striking interpretation: adjacent somatic cells can apparently invade and destroy the oocyte without earlier oocyte death or damage signals.

The recognition of this inducible developmental program of oocyte destruction has several implications beyond the immediate control of reproduction. That the different modes of induction, for example starvation and RAP treatment, result in different outcomes is suggestive of unique evolutionary strategies to control oogenesis in either a food- or natural antibiotic-dependent manner. In flies, these data support a hypothesis in which the establishment and maintenance of immunophilin (FKBP12, etc.) function in the genome would confer a significant reproductive advantage when molecules such as RAP are encountered, by preventing embryo laying in the short term, until an environment that will support larval development is encountered. Last, the conservation of this inducible mode of oocyte destruction in mammals suggests that the loss of immature oocytes during post-natal life may not only partly depend on intrinsic oocyte 'quality' (genetic/epigenetic or cytoplasmic integrity), but also may be controlled by the rate that somatic cell destruction of adjacent oocytes is induced. Future efforts to screen for genes required for mid-stage ISOD onset may reveal a large assortment of triggers and responsive genes that integrate environmental information to control fertility in mice as well as flies.

Our data support a new direction for efforts to protect fertility: blocking somatic cell destruction of oocytes. This mechanism warrants further study as the signaling network between the induction of ISOD through RAP or starvation and the execution of egg chamber destruction is essentially unknown.

\section{Materials and Methods}

Fly stocks and genetics. All fly strains are from the Bloomington Stock Center unless otherwise mentioned. The following fly lines were used for characterization, embryo laying, and offspring analysis: $y 1, w^{*}, m e r^{4}$, P\{neoFRT\}19A/FM7i, P\{ActGFP\}JMR ${ }^{3} ; \mathrm{w}^{*} ; \mathrm{mer}^{3}, \mathrm{P}\left\{\right.$ neoFRT\}19A/FM ${ }^{6} ; \mathrm{dlg}^{\text {hf }} / \mathrm{FM}^{\dagger}$ (Scott Goode); dlglv55 /FM7 (Scott Goode); w-; P $\{\mathrm{w}+$, vasP-Me31B-GFP $\}$ (Lynn Cooley); w-; dTor ${ }^{\mathrm{P}}$ /FRT40A/CyO (Thomas Neufeld); $\mathrm{st}^{1}{ }^{1}$ in $^{1}{ }^{1}, \mathrm{kni}^{\mathrm{ri}-1}, \mathrm{pp}$, $\mathrm{wts}^{3-17} / \mathrm{TM} 3$, Sb ${ }^{1}$; D4C drpr (-d5) rec8/TM3 ftz-lacZ; Roe ${ }^{1}$, pp; $\mathrm{y}^{1} \mathrm{w}^{*}$; I(2)gl4/CyO $y+; P\left\{\right.$ GawB\}185y, $w^{1118} ; w^{1118}, P\{$ GawB\}c204/TM3, Ser'; P\{UAS-IR.GFPFK506-bp2\}1, $y^{1} w^{*} . w^{1118} ;$ pVasa-me31B-GFP flies were found to undergo normal oocyte development and to have egg laying characteristics that did not significantly differ from the wild-type Canton-S strain.

Starvation, fly food supplementation, and embryo laying assessment. Agar plates containing grape juice have been used to mimic starvation conditions by other groups. We use hydrated agar containing no juice or other additives so that no energy or amino-acid sources are available in which 'starvation' is indicated. As flies lay embryos that can survive to adulthood (albeit at a greatly reduced rate) on grape juice agar, we refer to that treatment as 'partial starvation.' RAP experiments were performed using 'rich food,' grape juice agar plates supplemented with yeast. Concentrations of RAP were determined to be that of the volume of yeast slurry plus the volume of the juice agar within the collection plate $(2.5 \mathrm{ml})$. Plates were changed daily with fresh aliquots of RAP mixed within a yeast slurry. Embryo laying experiments were performed at $25^{\circ} \mathrm{C}$ except for dlghf flies whose embryo laying was tested at $18^{\circ} \mathrm{C}$ as well as $25^{\circ} \mathrm{C}$. In brief, five virgin females of each genotype were collected and crossed to three wild-type males. Either Canton-S or $\mathrm{w}-, \mathrm{P}\{\mathrm{w}+$, vasP-Me31B-GFP $\}$ males were used with no difference in subsequent egg laying seen between paternal strains. Flies were put into a cage with a collection plate having $250 \mu \mathrm{l}$ of a $25 \%$ yeast slurry with vehicle or RAP (LC Laboratories, Woburn, MA, USA) spread evenly over the surface of the collection plate. After confirming that male function was unaffected by RAP ingestion, gross assessment of embryo cellularization was performed for each experiment using light microscopic evaluation. Fertilization and early embryonic development were found to be unaffected by maternal RAP ingestion in this manner. For all reported parameters, each strain was tested in at least three separate trials.

Analysis of RAP uptake by flies. When 100 females either housed on vehicle or food containing the highest dose, $20 \mu \mathrm{M}$ RAP, for $72 \mathrm{~h}$, zero RAP was detected in vehicle-treated flies and $90 \mathrm{ng} \mathrm{RAP} / \mathrm{mg}$ wet weight of flies was detected in the RAP group. Rapamycin content was determined by GC/MS (Rocky Mountain Laboratories, Fort Collins, CO, USA). As individual flies weigh approximately $1.2 \mathrm{mg}$, each fly contains approximately $108 \mathrm{ng}$ RAP when fed the drug at this dose for this time period.

Western blot analysis. For analysis of intracellular proteins, cells were lysed using $1 \%$ NP40 and $0.1 \%$ SDS in the presence of protease inhibitors. Protein concentrations were calculated by BCA assay (Pierce Biotechnology, Rockford, IL, USA). A total of $20 \mu \mathrm{g}$ of each protein lysate were diluted in sample buffer and boiled for $5 \mathrm{~min}$. Proteins were resolved under reducing conditions on either 10 or $12 \%$ SDS-PAGE gels and then transferred onto nitrocellulose membranes (NEN Life Sciences, Boston, MA, USA). Membranes were blocked at room temperature for $1 \mathrm{~h}$ with $5 \%$ powdered milk in PBS $/ 0.05 \%$ Tween 20 (PBS-T). After three washes for 10 min, each with PBS-T, membranes were incubated overnight at $4^{\circ} \mathrm{C}$ with primary antibody (Rabbit anti-phospho p70 S6 kinase Thr389 at 1/1000, Cell Signalling; and mouse anti- $\beta$-actin $(1: 10000)$ (Sigma, St. Louis, MO, USA) in PBS-T/1\% FFPM. After this incubation, membranes were washed three times as before and then incubated at room temperature for $1 \mathrm{~h}$ with the appropriate secondary antibody conjugated to peroxidase $(1: 10000$, Southern Biotech, Birmingham, AL, USA) in PBS-T/1\% milk. After three washes for 10 min each with PBS-T and three washes for $10 \mathrm{~min}$ each with distilled water, the peroxidase-conjugated antibody was detected by enhanced chemiluminescence (Perkin Elmer, Waltham, MA, USA). $\beta$-actin was used as internal control, in addition to Ponceau Red, to validate transfer quality and the amount of protein loaded onto the gels.

Fluorescence visualization and immunostaining. Ovaries from various fly strains and experiments were dissected in PBS, fixed in devitellinizing buffer with $5 \%$ formaldehyde (methanol free to preserve GFP activity), under heptane for $5 \mathrm{~min}$. After fixation, ovaries were washed several times with PBT 
(PBS $+0.1 \%$ Tween-20) over $2 \mathrm{~h}$ after which the ovaries were dissected by pipetting up and down into a $200 \mu$ l pipet tip.

For visualization of DNA and actin (in combination with transgenic GFP), Hoescht dye $(0.2 \mu \mathrm{g} / \mathrm{ml})$ and Alexa Fluor 555 -conjugated phalloidin (Invitrogen, Carlsbad, CA, USA; per manufacturer's instructions) were used.

For whole-mount immunostaining, ovaries were then blocked in PBT-B (PBT + $2 \%$ bovine serum albumin) for $4 \mathrm{~h}$ at room temperature or overnight at $4{ }^{\circ} \mathrm{C}$. Ovaries were incubated with primary antibodies (mouse anti-FKBP12, BD Biosciences, San Jose, CA, USA; rabbit anti-PHH3 Ser10, Cell Signaling Technologies; mouse anti-Armadillo, Developmental Studies Hybridoma Bank/U. of lowa) for $12-16 \mathrm{~h}$ at $4^{\circ} \mathrm{C}$, diluted in PBT-B. Ovaries were then washed for $1.5 \mathrm{~h}$ with 10 changes of PBT, and then probed with labeled secondary antibodies (Alexa Fluor 488-, 568-, or 633-conjugated goat anti-rabbit or mouse) diluted $1 / 500$ in PBT-B plus $2 \%$ normal goat serum and incubated for $2 \mathrm{~h}$ at room temperature (in the dark).

Ovaries were then washed as above, except during the first wash $(15 \mathrm{~min})$ Hoechst $(0.2 \mu \mathrm{g} / \mathrm{ml}$, Invitrogen) and Alexa Fluor (488 or 555$)$ phalloidin was added. Ovaries were mounted in Prolong Gold (Invitrogen) and visualized using a Leica TCS SP5 confocal microscope.

\section{Conflict of interest}

The authors declare no conflict of interest.

Acknowledgements. We thank Drs Lynn Cooley, Marc Freeman, Paul Lasko, and Haifan Lin for scientific and editorial critiques of the manuscript while in preparation. Mentoring by Drs Charles Lockwood, Richard Hochberg, and Beth Schacter was important throughout the studies. Special thanks to Dr. Stuart Schreiber for criticism of implications for immunophilin evolution and function. Jimmy, Kevin Hom, Aylin Yaba, and James Yu are acknowledged for technical contributions to the studies. $\mathrm{JJ}$ is supported by Yale Department of Obstetrics, Gynecology, and Reproductive Sciences Research Funds and the Donaghue Yale Women's Health Research Program. This work is dedicated to the memory of Jacqueline Marie Sullivan.

1. Pepling ME, Spradling AC. Mouse ovarian germ cell cysts undergo programmed breakdown to form primordial follicles. Dev Biol 2001; 234: 339-351.

2. Pepling ME. From primordial germ cell to primordial follicle: mammalian female germ cell development. Genesis 2006; 44: 622-632.

3. Noce T, Okamoto-Ito S, Tsunekawa N. Vasa homolog genes in mammalian germ cell development. Cell Struct Funct 2001; 26: 131-136.

4. Baskind HA, Na L, Ma Q, Patel MP, Geenen DL, Wang QT. Functional conservation of asxl2, a murine homolog for the Drosophila enhancer of trithorax and polycomb group gene asx. PLoS One 2009; 4: e4750.

5. Mazzalupo S, Cooley L. Illuminating the role of caspases during Drosophila oogenesis. Cell Death Differ 2006; 13: 1950-1959.

6. Tilly JL, Kowalski KI, Johnson AL, Hsueh AJ. Involvement of apoptosis in ovarian follicular atresia and postovulatory regression. Endocrinology 1991; 129: 2799-2801.

7. Tilly JL. Molecular and genetic basis of normal and toxicant-induced apoptosis in female germ cells. Toxicol Lett 1998; 102-103: 497-501.

8. Lobascio AM, Klinger FG, Scaldaferri ML, Farini D, De Felici M. Analysis of programmed cell death in mouse fetal oocytes. Reproduction 2007; 134: 241-252.

9. Escobar ML, Echeverra OM, Ortz R, Vzquez-Nin GH. Combined apoptosis and autophagy, the process that eliminates the oocytes of atretic follicles in immature rats. Apoptosis 2008 13: $1253-1266$.

10. Inoue $\mathrm{S}$, Watanabe $\mathrm{H}$, Saito $\mathrm{H}$, Hiroi M, Tonosaki A. Elimination of atretic follicles from the mouse ovary: a tem and immunohistochemical study in mice. J Anat 2000; 196 (Part 1): 103-110.

11. Drummond-Barbosa D, Spradling AC. Stem cells and their progeny respond to nutritional changes during Drosophila oogenesis. Dev Biol 2001; 231: 265-278.

12. LaFever L, Drummond-Barbosa D. Direct control of germline stem cell division and cyst growth by neural insulin in Drosophila. Science 2005; 309: 1071-1073.
13. Pritchett TL, Tanner EA, McCall K. Cracking open cell death in the Drosophila ovary. Apoptosis 2009; 14: 969-979.

14. Gingras AC, Raught B, Sonenberg N. Regulation of translation initiation by FRAP/mTOR. Genes Dev 2001: 15: 807-826.

15. Wiederrecht GJ, Sabers CJ, Brunn GJ, Martin MM, Dumont FJ, Abraham RT. Mechanism of action of rapamycin: new insights into the regulation of g1-phase progression in eukaryotic cells. Prog Cell Cycle Res 1995; 1: 53-71.

16. Wullschleger S, Loewith R, Hall MN. Tor signaling in growth and metabolism. Cell 2006; 124: 471-484.

17. Jacinto E, Loewith R, Schmidt A, Lin S, Regg M, Hall A et al. Mammalian TOR complex 2 controls the actin cytoskeleton and is rapamycin insensitive. Nat Cell Biol 2004; 6: 1122-1128.

18. Harrar $Y$, Bellini $C$, Faure JD. Fkbps: at the crossroads of folding and transduction. Trends Plant Sci 2001; 6: 426-431.

19. Zhang H, Stallock JP, Ng JC, Reinhard C, Neufeld TP. Regulation of cellular growth by the Drosophila target of rapamycin dTOR. Genes Dev 2000; 14: 2712-2724.

20. Prasad M, Jang AC, Starz-Gaiano M, Melani M, Montell DJ. A protocol for culturing Drosophila melanogaster stage 9 egg chambers for live imaging. Nat Protoc 2007; 2: 2467-2473.

21. Wang X, Proud CG. Nutrient control of TORC1, a cell-cycle regulator. Trends Cell Bio 2009; 19: 260-267.

22. Brand AH, Perrimon N. Targeted gene expression as a means of altering cell fates and generating dominant phenotypes. Development 1993; 118: 401-415.

23. Van Doren M, Williamson AL, Lehmann R. Regulation of zygotic gene expression in Drosophila primordial germ cells. Curr Biol 1998; 8: 243-246.

24. Kennerdell JR, Yamaguchi S, Carthew RW. RNAi is activated during Drosophila oocyte maturation in a manner dependent on aubergine and spindle-E. Genes Dev 2002; 16 : 1884-1889.

25. Zhang Y, Billington CJ, Pan D, Neufeld TP. Drosophila target of rapamycin kinase functions as a multimer. Genetics 2006; 172: 355-362.

26. Udan RS, Kango-Singh M, Nolo R, Tao C, Halder G. Hippo promotes proliferation arrest and apoptosis in the Salvador/Warts pathway. Nat Cell Biol 2003; 5: 914-920.

27. Polesello C, Tapon N. Salvador-warts-hippo signaling promotes Drosophila posterior follicle cell maturation downstream of notch. Curr Biol 2007: 17: 1864-1870.

28. Meignin C, Alvarez-Garcia I, Davis I, Palacios IM. The salvador-warts-hippo pathway is required for epithelial proliferation and axis specification in Drosophila. Curr Biol 2007; 17: 1871-1878.

29. Hamaratoglu F, Willecke M, Kango-Singh M, Nolo R, Hyun E, Tao C et al. The tumoursuppressor genes NF2/Merlin and expanded act through hippo signalling to regulate cell proliferation and apoptosis. Nat Cell Biol 2006; 8: 27-36.

30. MacDougall N, Lad Y, Wilkie GS, Francis-Lang H, Sullivan W, Davis I. Merlin, the Drosophila homologue of neurofibromatosis-2, is specifically required in posterior follicle cells for axis formation in the oocyte. Development 2001; 128: 665-673.

31. LaJeunesse DR, McCartney BM, Fehon RG. Structural analysis of Drosophila merlin reveals functional domains important for growth control and subcellular localization. J Cell Biol 1998; 141: 1589-1599.

32. Zhao M, Szafranski P, Hall CA, Goode S. Basolateral junctions utilize warts signaling to control epithelial-mesenchymal transition and proliferation crucial for migration and invasion of Drosophila ovarian epithelial cells. Genetics 2008; 178: 1947-1971.

33. Assmat E, Bazellires E, Pallesi-Pocachard E, Le Bivic A, Massey-Harroche D. Polarity complex proteins. Biochim Biophys Acta 2008; 1778: 614-630.

34. MacDonald JM, Beach MG, Porpiglia E, Sheehan AE, Watts RJ, Freeman MR. The Drosophila cell corpse engulfment receptor Draper mediates glial clearance of severed axons. Neuron 2006; 50: 869-881.

35. Li W, Baker NE. Engulfment is required for cell competition. Cell 2007; 129: 1215-1225.

36. Oldham S, Montagne J, Radimerski T, Thomas G, Hafen E. Genetic and biochemical characterization of dTOR, the Drosophila homolog of the target of rapamycin. Genes Dev 2000; 14: 2689-2694

37. Yaba A, Bianchi V, Borini A, Johnson J. A putative mitotic checkpoint dependent on mTOR function controls cell proliferation and survival in ovarian granulosa cells. Reprod Sci2008; 15: $128-138$

38. Huang JH, Rajkovic A, Szafranski P, Ochsner S, Richards J, Goode S. Expression of Drosophila neoplastic tumor suppressor genes discs large, scribble, and lethal giant larvae in the mammalian ovary. Gene Expr Patterns 2003; 3: 3-11.

39. Sarbassov DD, Ali SM, Sengupta S, Sheen JH, Hsu PP, Bagley AF et al. Prolonged rapamycin treatment inhibits mtorc2 assembly and akt/pkb. Mol Cell 2006; 22: 159-168.

40. Mirouse V, Swick LL, Kazgan N, St Johnston D, Brenman JE. LKB1 and AMPK maintain epithelial cell polarity under energetic stress. J Cell Biol 2007; 177: 387-392.

\section{Supplementary Information accompanies the paper on Cell Death and Differentiation website (http://www.nature.com/cdd)}

